\title{
artículos
}

\section{Las tareas de la fundamentación de la bibliotecología}

\author{
Miguel Ángel Rendón Rojas \\ InvestigadordelCen tro Universitario de Investigat \\ cionesBibliotecológicas. UNAM. \\ Cir cui to In te rior, Torre II de Hu ma ni da des Piso 12 , \\ Ciu dad Uni ver si ta ria, C.P. 04510. Te1.623-03-43. \\ e-mailmarr@servidor.unam.mx
}

\section{RESUMEN}

$E$ n el presente ar tícu lose ex ponen los diversos as pectosa trabajarparafunda mentar la Biblio te co lo gía como cien cia. Di chos as pec tos son el fi losófi co, el me to do ló gi co, el teó ri co, el ló gico y el ex tra ló gi co. En el pri me ro se pro po ne elstatus on to ló gi co rea lis ta del que se par te, y los proble mas gno seo ló gi co, an tro po ló gi co, éti co y axio ló gi co a re sol ver; en el ni velmetodológico se plan tea la ne ce si dad de pre ci sar los mé to dos que uti li za la Bi blio te co logía; en los ni ve les teóri coy ló gi co es ne ce sa riocla rifi car, pre ci sar y sis te ma ti zar el apa ra to con ceptual y las le yes biblio te co lógi cas; y, por úl ti mo, en la fun da men ta ción ex tra ló gi ca, esin dis pen sable de terminar las herramientas (conceptos, leyes, métodos) importadas de otras disciplinas y establecer si cam bian o no su ca rác ter en la nue va área.

\section{ABSTRACT}

$T$ his ar tic le sets forth se ve ral as pects that need to be wor ked on for the es ta blish ment ofbibliothecology as a science; among them, tho se of a philo sophi cal, methodologi cal, logical and extralogical nature. The first one posits a realistic ontological status as starting point and the gnoseological, anthropological, ethicaland axiologi cal problems to be solved. The n eed to determine the methods employed in Bibliothecology is posed at a methodological level. Within the theo re ti cal and lo gi cal do mains it is ne ces sary to cla rify, spe cify and sys te ma tize the concep tual appa ra tus and bi blio the cologi cal laws. Lastly, in the case of ex tra logi cal foundationsit is essen tial to de ter mi ne tho se tools im por ted from other dis ci pli nes (con cepts, laws, m ethods) and es ta blish whether their cha rac ter chan ges or not in their new do main.

\section{INTRODUCCIÓN}

$\mathrm{U}$ na dis ci pli na pue de de sa rro llar se de una maneraex tensiva acumulandodatosempíricos y creando conceptos, teorías, leyes y prin ci pios para un gru po o gru pos de fe nó menos - o for man do téc ni cas para re sol ver problemas que se le presentan- solucionesque no sólo res pon den a ca sos con cre tos, sino que pueden ser vir para algo muy ge ne ral.

En un mo men to de su de sa rro llo his tórico, sin em bar go, ese proce so acu mu la ti vo noes su fi ciente para satisfacer el ulterior avance de la cien cia. Nace en ton ces la ne ce si dad de or denar, je rar qui zar e in te rre la cio nar todo ese conocimiento ob te ni do para que di cha dis ci plina se constituya en un sistema capaz de ex plicarcoheren te men te, en su to ta li dad y no fragmentariamente, los fenómenos para los que fue crea da y, asi mis mo, para que se pongan de ma ni fies to to das las re la cio nes e in terrelaciones entre conceptos, principios y leyes que subyacen implícitas en esas técnicas creadas parasatisfacernecesidades prácticas.

Más aún, ante la cantidad de datos acumulados, sur ge la in quie tud de vol ver la vis ta ha cia la mis ma cien cia y cues tio nar se so bre ella. Es en este momento cuando se puede decir que se ha alcanzado un grado de madurez suficiente como para reflexionar sobre ella misma.

Antes se investigaban los fenómenos que se tenían y debían conocer, en resolver tareas que se presentaban, pero ahora la investigación se vuelve sobre la ciencia. Se hace un cuestionamiento sobre su propia identidad, so bre sus lími tes y so bre sus fi na li da des y sus me dios. Es decir, apa re ce la necesi dad de fundamentar esa disciplina, de hacer objeto de estudio a la misma ciencia, o sea, de hacer metaciencia. Este fenómeno no es tanto una crisis in terna, comosín to ma de cre ci mien to y madurez.

Una si tua ción si mi lar la po de mos ob servarno sólo en el de sa rro llo de las cien cias, sino también en el de sa rrollo de la per so na li dad hu mana. La psi co lo gía evo lu ti va nos ex pli ca cómo, después de un largo periodo de crecimiento, cuan do el ser hu ma no ha al can za do cier ta ma- 
du rez, en la adoles cen cia, el jo ven se em pieza a cuestionar so bre su pro pia iden ti dad; su yo lo contrapone al no-yo para saber quién es, qué lugar ocupa en este mundo y cómo relacionarse con él.

Un claro ejemplo de este fenómeno, relacionado con el desarrollo de las ciencias, lo pode mos ob servar en la his to ria de las Ma te máticas. En la segunda mitad del siglo pasado, cuan do des pués de más de dos mi le nios de desenvolvimiento, esta ciencia había realizado asombrosos avances en áreas como la Aritmética, el Cálculo Integral y Diferencial, el Álgebra y la Trigonometría, entre otras, los matemáticos se empiezan a preocupar por fun da men tar sucien cia. El ma te máti co y filó so fo ale mán G. Fre ge de sa rrolló un pro gra ma para cum plir esta ta rea: se preo cu pó por de finir y aclarar conceptos tales como axioma, variable, número, clase, deducción o de mostración y otros. Para esto creó un len guaje for mal nue vo que fue el cál cu lo ló gi co matemático y analizó con ese instrumento el contenido de las matemáticas, es decir, por primera vez hizo Metamatemáticas. ${ }^{1}$ Sin em bargo, dado que el ob je ti vo per se gui do por Fre ge no fue al can zado, otros cien tíficos ta les como B. Russell, A. Whi tehead, G. Pea no D. Hil bert, L. E. Brou wery A. Heyting con ti nuaron con esa tarea. ${ }^{2}$

Un fe nó me no si mi larocu rriócon la Física a partirde fi nes del si glo pasa do y princi pios de éste, ante el nacimiento de la Física de la relatividad y Física cuán ti ca. El avan ce de la cien cia si guió su cur so, pero los cien tí fi cos se cuestionaron sobre el nuevo significado que adquirían conceptos que tradicionalmente manejaban, tales como materia, causalidad, sujeto y objeto de la investigación, tiempo y espacio, entre otros. Los físicos manejaban esos términos con base en fórmulas y modelos matemáticos dentro de su ciencia; empeza ron a re fle xio nar so bre esos mo de los y so- bre los métodos utilizados en su quehacer científico; empezaron a hacer filosofía de la Física y así aparecieron los trabajos de E. Mach, H. Re in chen bach, R. Car nap y O. Neut rath en los que se bus caban los prin ci pios filosóficos que funda men taran la Física. ${ }^{3}$

Si estas dos cien cias con una his to ria tan larga, después de tantos siglos de desarrollo se cuestionaron sobre sus fundamentos, la $\mathrm{Bi}$ bliotecología, que tiene una historia mucho más re cien te, pue de ha cer lo sin in ti mi dar se.

\section{DIFERENTES ASPECTOS DE LA FUNDAMENTACIÓN DE UNA CIENCIA}

Mu cho se ha habla do y es cri to so bre la fundamen tación de la Biblio te co logía,${ }^{4}$ mas es nece sa rio es pe cifi car en quécon sis te di chafundamentación y qué tareas hay que realizar para lle var la a cabo.

La fundamentación de una disciplina abarca diversos aspectos que muchas veces no se aprecian. Se debe realizar una fundamentación filosófica, una fundamentación lógica, una fundamentación metodológica, una fundamentación del cuerpo teórico y una fundamen ta ción ex tra teórica. Esto no im pli ca de cir que sean pa sos que se de ban efec tuar con una secuencia de ter minada, sino que son as pectos de un todo que se complementan e interrelacio nan, de pen dien do unas de otras; si se rea lizan unas, se es ta rán rea li zan do otras. Nues tro objetivo final será encontrar las respuestas a las preguntas ¿qué es esa cien cia? ¿qué es tudia? ¿c ómo lo es tu dia? ¿p ara qué lo es tu dia?

La fun da men ta ción fi lo só fi ca con sis te en utilizarcategorías, principios, teo rías de la fi loso fía para la so lu ción de pro ble mas on to ló gicos, gnoseológicos, antropológicos, axiológicos y éticos de la disciplina analizada. Estos fundamentos filosóficos dependen de una concepción filosófica y deben de ser ca pa ces de es ti mu lar la evo lu ción de la cien- cia, dar una ex pli ca ción de su exis ten cia, es timular su desarrollo y coadyuvar a su utilización práctica.

Como con se cuen cia de la fun da men ta ción filosófica se debe tener una fundamentación gnoseológica, esto es: justificar la existencia del ob je to de la teo ría en la rea li dad y es pe cificar el enfoque particular que se estudia de él; como di rían al gu nos fi ló so fos, de ter mi nar elobje to quod y quo de la disciplina. ${ }^{5}$ La ciencia tie ne como ob je ti vo re fle jar la rea li dad (no de una ma ne ra ab solu ta, sino con cier to gra do de pro fun di dad que se irá ahon dan do a me dida que la cien cia se de sa rro 1la), es de cir, debe de haber una adecuación de la teoría con la realidad, lo que significa que los enunciados de la dis ci pli na se rán ver da de ros y esa ver dad pro por cio na rá una ex pli ca ción del tro zo de la realidad que estudia, debe hacerla más comprensible, ayudarnos a predecir y controlar los fenómenos estudiados.

De acuer do con la na tu ra le za del ob je to que se estudia hay que buscar una fundamentación me to dológica; esnecesariopreci sarlos métodos usa dos para cons truir y de sa rro llar la disci plina. De ter mi nar si se usa rán mé to dos empíricos, estadísticos, de duc tivosode análisis, dependiendo del objeto estudiado y del que más se adecue para lle gar a la verdad.

Por otro lado, la fundamentación del cuerpo teórico de la disciplina consiste en analizar, esclarecer y precisar los conceptos fundamen ta les de di cha teo ría, es pe cificar sus le yes y principios, hacer obvias las teorías en las que se ba san cier tas téc ni cas uti li za das confines prácticos.

Posteriormente o simultáneamente se debe llevar a cabo la fundamentación lógica que significadeterminar las relaciones existentes entre los elementos ya analizados, clarificados y pre ci sa dos del cuer po teó ri co de la disciplina. Una relación primordial entre los enunciados de una teoría debe ser su consisten cia, o sea, que no ha brá con tra dic ción en tre

1 - Frege, Gottlob. Conceptografía. Un lenguaje de fórmulas semejante al de la Aritmética para el pensamiento puro. Los fundamentos de la Aritmética. México: Unam, 1972.

2 - Rusell, B. y Whitehead, A. Principia Mathematica. Madrid: Paraninfo, 1981. - Hilbert, D. y Bernays, P. Grundlagen der Mathematik. Berlin: J. Springer, 1934. - Heyting, A. Introducción al Intuicionismo. Madrid: Tecnos, 1976.

3 - Carnap, R. Fundamentación lógica de la Física. Buenos Aires: Sudamericana, 1969. - Mach, E. Knowledge and error. Holland: D. Reidel, 1976. - Reichenbach, H. The philosophy of space \& tine. New York: Dover, 1958.

4 Así por ejemplo J. Shera observa que sólo cuando el proceso bibliotecario sea un sistema con un cuerpo teórico determinado y el servicio bibliotecario sea el fruto de un conocimiento debidamente fundamentado será cuando se pueda hablar de que la Bibliotecología obtuvo la cualidad de ciencia con derecho propio.

- Shera, J. Knowing books and men; Knowing computers, too. Littleton: Libraries Unlimited, 1973. p. 9.

También Deyanira Sequeira expresa la misma necesidad al plantear el problema de que "Hace falta buscar cuáles son las bases epistemológicas de la profesión [de la Bibliotecología, M.A.R.]..."

- Sequeira Ortiz, Deyanira. La Bibliotecología como ciencia. San José, Costa Rica: Publitex, 1988. p. 9.

5 La fundamentación gnoseológica es una consecuencia de la fundamentación filosófica puesto que nuestra gnoseología depende directamente de la posición ontológica adoptada. Si partimos de una ontología dialéctica, nuestra gnoseología también será dialéctica; si la primera es idealista, la segunda también lo será. 
ellos. Esta con sis ten cia pue de ser se mán ti ca y sin tác ti ca en el caso de las teo rías for ma li zadas. En el primer caso, se encontrarán dos proposiciones ta les, que una afir me una cosa so bre un ob je to, y otra que, si mul tá nea men te so bre el mis mo as pec to y so bre el mis mo objeto, lo niegue. En el caso de la consistencia sin tác ti ca no se dará una si tua ción tal que una proposición sea teo re ma (esté de mos trada)en esa teoría y al mismo tiempo sea teorema su negación.

Pero la con sis ten cia es sólo una re la ción ló gica que debe darse den tro de la teo ría (si bien es cierto esencial y necesaria) para que se pueda hablar de disciplina científica. Dentro de la fundamentación ló gi ca se bus ca rá también qué con cep tos y le yes son in de pen dientes, o sea, que sir ven como prin ci pios para gene rar otros con cep tos y le yes, sin de pen der a su vez de otros. Se debe esclarecer cómo se deduce, a partir de esos principios, el cuerpo teórico de la dis ci plina, qué prin ci pios ex traló gi cos se uti li zan para que la es truc tu ra de la teo ría tome un ca rác ter sis te má ti co. Se en contrará la lógica de la teoría, las relaciones de independencia, subordinación, compatibilidad e identidad entre los enunciados. Esta operación es la complementación de la anterior, la síntesis del análisis.

Asimismo, es indispensable poner de manifiesto lafunda men ta ción ex trateórica, estoes, analizar el lenguaje, los principios, leyes y mé to dos de otra teo ría que se usan para re solver los problemas de la disciplina estudiada. Es nece sa rio de ter mi nar cómo se em plean los instrumentos exportados de otras áreas, si man tie nen el mis mo sen ti do o al uti li zar se en un cam poes pecífi co la con no ta ción se mán ti cacambia.

De una manera abstracta, se puede de cirque lo pri me ro es de ter mi nar el ob je to de es tu dio. Por supuesto que eso sería lo ideal, pero muchas ve ces, eso que de be ría ser lo pri me ro, es loúl ti mo; resul ta do del es tu dio rea li za do preli mi nar men te. Así, por ejem plo, en el caso espe cífi co de la fun da men ta ción de la Bi blio tecología, R. La fuen te ex presa la nece sidad de una síntesis del conocimiento bibliotecario (lo que en nuestra terminología equivale a realizar lafun da men tación teórica y ló gica,es decir, reunir, aclarar, ordenar, evaluar, encadenar y sintetizar el cuerpo teórico de la $\mathrm{Bi}$ bliotecología, utilizando como elemento extra lógi cola ex plicación teleológica) y a partir de él encontrar el objeto y método de la Bibliotecología ${ }^{6}$ (fundamentación filosófica y metodológica). Otroenfoque es el de S. San-

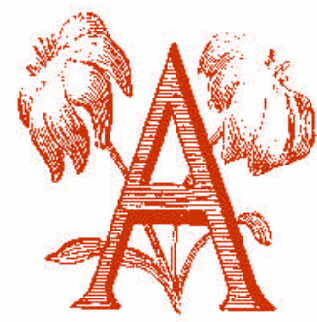

der que exige el análisis his tórico de diferentes con cep cio nes biblio te co ló gi cas para que a par tir de él se en cuen tren las teo rías, los conceptos, las leyes de la Bibliotecología y con base en ellos se de ter mi ne el ob je to de es tu dio de estadis ciplina. ${ }^{7}$

\section{DIFERENTES ASPECTOS EN LA FUNDAMENTACIÓN DE LA BIBLIOTECOLOGÍA}

Por lo tanto, de acuerdo a nuestro plan establecido anteriormente, para la fundamentación de la Bi blio te co lo gía, las ta reas a rea li zar se rían las si guien tes:

\section{Fundamentación filosófica}

Consideramosnecesario par tir de una pre misa on to ló gi ca que es tablezca la exis ten ciareal del mun do circun dan te y la ca pa ci dad del suje to para co no cer lo. Elser es lo pri me ro, pero el ser en sí no exis te, sólo es, lo que exis te son entes concretos con un ser determinado por una esen cia, la unión de la esen cia y el ser nos da el ente, que es lo que se nos pre sen ta y toda la acción encaminada (dirigida) a esos entes estará en función de su ser específico. Esto nos ayu da rá a ade cuar los mé to dos de es tu dio de una manera objetiva, si prescindimos de esta premisa, caeremos en el subjetivismo me to dológico (anarquis mo), ya que en tonces es el sujeto (investigador) el que a su juicio utilizará el método que más le parezca útil y se gún élle ofrez ca me jo res re sul ta dos. Peroel hecho de que exista diversidad de métodos, no sig ni fi ca que no haya uni dad, que haya sola mente un convencionalis mome to dológico. Esta uni dad está dada por la na tu ra le za del obje to es tu dia do. Si un mé to do re sul ta ade cua do es por que el ser del ob je to exi ge ese mé to do, no es porque de una forma arbitraria se haya creado ese método y el objeto de estudio se haya so me ti do a él, al con tra rio, el mé to do se creó con base en el ob je to. Si se pier de de vista la prioridad ontológica, se forma un caos, incluso, la verdad y las leyes científicas se pueden definir con base en situaciones cambiantes como son los paradigmas establecidos por la comunidad científica, los resultados pragmáticos obtenidos o los cánones es ta ble ci dos por los gru pos de po der. Pero no debe olvi dar se que esos pa ra dig mas, esos resultados o cánones tie nen va lor no en sí mismos, sino por que se ade cuan a la rea li dad estu dia da. La re la ción no es: si una teo ría es útil, entonces es verdadera, sino si una teoría es verdadera,entonces es útil.

Ahora bien, la práctica se puede entender como un criterio úl ti mo de ver dad, pero des-

6 - Lafuente, R. "La síntesis crítica del conocimiento bibliotecológico: su valor para la investigación bibliotecológica." En Investigación bibliotecológica. Enero-junio 1988. v. 3, n. 6. p. 3-10.

7 - Sander, S. "Los elementos histórico-teóricos para la indagación de la estructura teórica de la Bibliotecología." En Investigación bibliotecológica. Enero-junio 1989. v. 3, n. 6. p. 31-37. 
pués de la ob serva ción, ex pe ri men ta ción, veri fi ca ción; del em pleo de los mé to dos de ductivos, inductivos, estadísticos y otros, la práctica no tomada de una manera subjetiva (como en el prag ma tis mo en don de se le identifica con la utilidad), sino como un proceso objetivo de transformación y asimilación de la realidad, no individual sino colectivo, social e histórico. Más aún, la práctica se emplea para fal sear la teo ría, como se in fie re de la implicación ex presa da an te rior mente: sila ver dad de una teo ría im pli ca su uti li dad y si en la prác ti ca se com pro bó que no es útil, en tonces pormodustollens ob te ne mos que esa teoría no es verdadera.

La pre mi sa de la pri ma cía del ser tam bién nos evitará caer en el absolutismo metodológico, que es propio del positivismo. No es posible que to das las cien cias uti li cen el mis mo mé todo debido precisamente a que el ser de cada objeto está determinado por diferentes esencias que los ha cen di fe ren tes, por lo que se rán estudiados de diversasformas. ${ }^{8}$

Esta pro pues ta on to ló gi ca nos obli ga ráa de fi nir nos con res pec to a lo que es la bi blio te ca, el libro y el usuario; por lo que la selección de ma te riales, su cla sificación y ca ta lo ga ción ya no serán una actividad subjetiva y arbitraria, sino ob je ti va con base en las ca rac te rís ti cas de esos tres componentes fundamentales de la Bibliotecología, así como también las leyes bibliotecológicas tendrán un carácter objetivo. En el campo axiológico se tomarán en cuenta los valores que se desean alcanzar en función al enfoque que se le dé al usuario, es necesario determinar si se le considera Zoon politicon, Homo sapiens, Homo faber (o tal vez Homohabilis), un con jun to de re la ciones so cia les, un ser crea do a ima gen y se me jan za de Dios, un ser en- sí cons cien te de es tar conde na do a ser li bre y de sean do li be rar se de esa li ber tad, o de otra for ma, para que en fun ción de esa con cep ción se or ga ni ce nues tro quehacer bibliotecológico.

Si nues tra ac ción va en ca mi na da a de sa rrollar el ser del usua rio como lo con ce bi mos, en tonces es un va lor, si de gra da o fre na su de sa rrollo, es un an tiva lor. Ésta es una ta rea esen cial para fundamentarlaBibliotecología, se debe realizarunatareafilosófica-antropológicade análi sis del usua rio, o si se de sea ex por tar la de una concepción filosófica determinada, cosa muy difícil, por que cada una ab solu ti za su posición, más bien, se ría una sín te sis dia léc ti ca (no ecléc ti ca) de al gu nas de ellas. Cla ro está, aquí siem pre se ten drán di ver gen cias, de bi do a que las escuelas filosóficas nunca se han pues to de acuer do, pero es in dis pen sable ocupar una posición lógicamente consistente, compatible con el conocimiento científico y la realidad, y que nos ayude a desarrollar nuestra disciplina (es interesante señalar cómo la Bibliotecología soviética-rusa, en este punto, parte de un supuesto que le ha dado unidad - la concepción marxista - lo que le ha per mi ti do de sa rrollar se como es cuela y obtener importantes resultados).

En el cam pogno seo ló gi co es ne cesario de termi nar el ob je to de es tu dio de la Bi blio te co logía, iden ti fi car los pro ble mas a los que se va a dedicar, delimitar su campo de acción, responder a las siguientes preguntas ¿la Bibliote cología es cien cia, téc nica o tec no logía? ¿es una rama de la Cien cia o Cien cias de la In forma ción? ¿cuáles su re la ción con la Bi blio grafología, la Informática (en el sentido de Mijailov), la Archivología, la Museología? ¿la Bibliotecología tiene puntos de contacto con las Cien cias de la Co mu ni ca ción, la Teo ría de la Información y la Cibernética? ¿qué es lo que rige la diversidad y la unidad en la especia li za ción de las cien cias?

En ocasiones se utiliza el término epistemología para designar la investigación y fundamentación del conocimiento científico, dejando a la gnoseología la tarea de analizar el conocimentoengeneral. ${ }^{9}$ De bi do a que el conocimiento científico se caracteriza por su grado de generalidad, uni versali dad y necesidad ob te ni do de una mane ra me tó dica, en tonces una fundamentación epistemológica abarcaría, aparte de la fundamentación gnoseológica, lo que nosotros hemos llamado fundamentación metodológica, fundamentación teóri ca y fun da men ta ción ló gi ca. He mos optado por diferenciar todas esas fundamentaciones, por razones metodológicas, para que de esta manera aparezcan más claras las ta reas que hay que rea li zar y po ner de re lie ve la re la ción es tre cha que tie ne la fun da men tación gno seo ló gi ca con la fi lo só fi ca, ya que la concepción ontológica de que partamos va a con di cio nar la gno seo lo gía o teo ría del co nocimien to que adop te mos.

\section{Fundamentación teórica}

El lenguaje de una disciplina ocupa un lugar muy im portan te, pues to que es la he rra mien ta con la cual se de sig na rán los con cep tos que la cons ti tu yen. Por lo tan to, se debe as pi rar a tener una ter mi no lo gía pro pia, su fi cien te y precisa. Podemos decir que la correcta formación, introducción y unificación de términos ayu da al ul te rior de sa rro llo de un cam po particular de conocimien to. Se de ben evi tar equi vocidad, tautologismo y relativismo, lo que nos evi ta rácaer en con fu siones, va gue da des, ambigüedades y en un vacío de sentido.

Por lo tanto, es necesario definir conceptos claves en Bibliotecología tales como información y conocimiento, flujo de información, necesidad de información, registro de información, documen to, usuario, biblioteca, centros de análisis y referencias, centros de información, clasificación, actividad bibliote caria; todo esto es ta ble cien do cla ra men te la especificidad que tienen estos conceptos en esta área del co no ci mien to y la di fe ren cia que tienen esos términos en otras disciplinas como la Filosofía, la Teoría de la Información, la Archivología, la Ciencia de la Información, la Pedagogía, el Pe riodis mo y otras.

Almis mo tiem po, es nece sa rio es pe cificar las teorías y leyes que subyacen en la actividad bibliotecaria, por ejemplo, los princi piosque rigen los diferentes tipos de clasificación bibliotecológica.

\section{Fundamentación extrateórica}

Es ne ce sario de ter mi nar qué con cep tos, téc nicas y mé to dos se to man de otras áreas de conocimiento; por ejemplo de la Cibernética y Teoría de la Información al automatizar el quehacerbiblio te cológico; de lapsicologíaal analizar la psicología del lector, sus motivaciones e in te re ses; de la so cio lo gía al es tu diar el en tor no so cioe co nó mi co del lec tor, los fac-

8 Se habla de diversidad de la naturaleza de los objetos en cuanto que son objetos de estudio, no en cuanto lo que son en sí. Así por ejemplo, la esencia de un tigre en cuanto tigre es muy diferente a la de un oso en cuanto oso, pero el método para estudiarlos puede coincidir porque ambos comparten la propiedad de ser seres vivos y la Biología los aborda desde ese punto de vista y los estudiará de una manera distinta a como lo haría la Psicología animal, si se acepta la existencia de esa disciplina o la Física, si es que le llegaran a interesar esos objetos y los tomara únicamente como cuerpos con determinado peso, volumen y sujetos a determinadas leyes físicas. Así también, seres tan disímiles como un cuerpo celeste: el sol, un isótopo de un elemento químico: el ozono y los seres vivos se estudian c onjuntamente por la Ecología porque se ven como elementos de una clase con una característica común. Por otro lado, un mismo objeto "el sol" p uede ser estudiado con diferentes métodos según la característica que se determine como esencial para su estudio: es un cuerpo celeste, un elemento del sistema ecológico o un objeto de culto religioso; en el primer caso se utilizarán modelos matemáticos exactos para calcular la fuerza de gravedad que ejerce sobre otros cuerpos celestes, pero la experimentación es muy difícil emplearla; en el segundo caso, la Matemática ya no es de gran ayuda, pero es posible utilizar la observación y hasta cierto punto la experimentación; en el último caso no se puede recurrir ni a las Matemáticas ni a la experimentación, sino a la investigación e interpretación de monumentos y otros documentos que testimonien el culto del sol en determinadas culturas.

9 - Bunge, M. Epistemología. Barcelona: Ariel, 1980. 
tores sociales y culturales que influyen en la organización del trabajo bibliotecológico.

Si en el análisis filosófico determinamos cómo se toma al usuario ontológicamente para, a par tir de ahí, es ta ble cer nues tra axio logía y obje ti vos fi na les muy ge ne ra les, y sien ladefiniciónteóricadeusuario de terminamos más particularmente qué entendemos por él cuando se acerca a pedir informaciónbibliotecológica, en este momento lo delimitamos más, co lo cán do lo y a como un usua rio con característicasespecíficas: es un estudiante, un niño, un in ves tigador, un políticou hombrede negocios; se determina su entorno social: vive en la ciudad, en una poblaciónpequeña, etcétera.

Otras dis ci pli nas tam bién apor tan sus co no cimientos a la Bibliotecología y se debe poner en cla ro, por ejem plo, cómo y en qué la Ad ministración nos ayu da a rea li zar nues tra ta rea; cómo la Ciencia Política nos puede orientar para ac tuar en con di cio nes po líti cas bien concre tas de una ma ne ra más efi caz. Tam bién la Pedagogía, la Lingüística y la Semiótica hacen sus aportaciones a la Bibliotecología, es necesarioestablecer cuáles y cómo.

\section{Fundamentación metodológica}

En este apartado el objetivo es analizar los mé to dos que se ade cuan al ob je to de es tu dio de la Bibliotecología, cuáles son y cómo se apli can. Se pue de dar el caso de que el ob je to de es tu dio no esté aún to tal men te de li mi ta do, entonces, por una parte, se debe de te ner una hipótesis de cuál es ese objeto de estudio, y por otra, analizar los métodos que históricamen te se han uti li za do y que han dado re sul tados positivos, infiriendo de esta manera, tomando en cuenta todos nuestros supuestos filosóficos, teóricos y extrateóricos, si nuestra hipótesis es correcta o, si no lo es, cambiarla.

En tre los prin ci pa les mé to dos a ana li zar, para determinar su aplicación en la Bibliotecología, te ne mos elló gi co-de duc ti vo que se utilizaparaclasificarycatalogar los documentos, obteniendo de esta manera un sistema con ciertas relaciones entre sus elementos. Se debees pecificarsies tas re laciones son anivel sintáctico (entre símbolos, independientemente de su contenido, aunque de éste partimos para simbo li zar los docu men tos) o a nivel se mán tico(relaciones en tre contenidos)o incluso a nivel pragmático (relaciones entre símbolos y usuarios) o si se dan todos estos niveles a la vez.

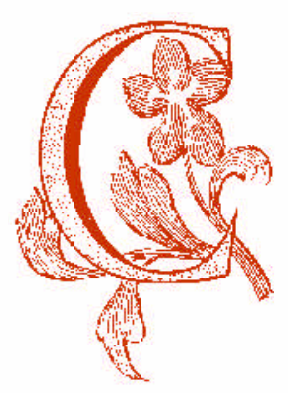

\section{"Otro método del que se sirve la Bibliotecología es el hermenéutico, empleado para la interpretación de documentos"}

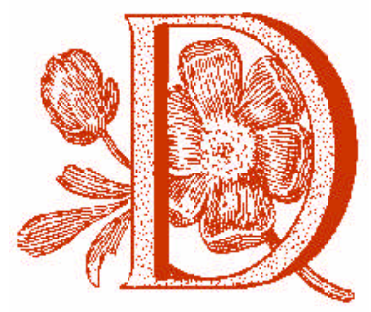

Cabe acla rar que en la Ló gi ca con tem po rá nea los niveles sintáctico y semántico tienen un gra do de de sa rro llo mu cho más alto que el nivel pragmático, el cual apenas están empezando a estudiar. Históricamente primero se buscó la formalización y axiomatización de sis te mas ló gi cos, des pués con los tra ba jos de A. Tarsky, la aten ción se vol vió ha cia los problemas semánticos y últimamente se están bus can do crear sis te mas en los cua les se ten ga un lu gar para el su je to que ha bía sido ex clui do de la Lógica. ${ }^{10}$

Otro mé to do del que se sir ve la Bi blio te co logía es el hermenéutico, empleado para la inter pre ta ción de docu men tos. Es necesarioestablecer cómo es que se garantiza la objetividad y fidelidad al analizar nuestros docu men tos cuan do se rea li zan cier tas ac ti vi dades bibliotecarias. Qué pasos y procedimien tos se de ben se guir y cuáles con di cio nes se de ben de ob servar para ob te ner el re sul ta do deseado. ${ }^{11}$

Asimis mo, ya que serealizan in vestigaciones de cam po y uti lizan mé to dos es ta dís ti cos para detectar necesidades de información, intereses de los usua rios, erro res en la or ga ni za ción del trabajo bibliotecario, etcétera, es indispen sable es ta ble cer las ba ses teó ricas de es tos métodos para que nuestro trabajo sea científico.

\section{Fundamentación lógica}

Enlafundamentación me to do lógicaseindicó que es necesario analizar la estructura de los sistemas bibliotecológicos de clasificación y en con trar qué tipo de re la cio nes exis tía en tre sus ele men tos, esto tam bién es par te de la fundamentación lógica y podríamos agregar semiótica.

Al mismo tiempo, se debe analizar cómo se relacionan los distintos conceptos entre sí. Por ejemplo, el contenido del concepto de información es más am plio, es tre cho o idéntico que el de conocimiento y si uno está inclui do en otro, en ton ces agre gan do cier tos datos ini cia les, de este úl ti mo se pue de de du cir ló gi ca men te el pri me ro. Así tam bién, del concepto de información, como más amplio, se pueden ir generando, por operaciones lógicas, con cep tos más li mi ta dos ta les como flu jo de información, necesidad de información y otros.

Por último, se mostrará la consistencia de la teoría bibliotecológica, que no haya contradic ciones en tre sus enun cia dos y si se tie ne la sos pe cha de que és tas tie nen lu gar, es ne ce sario es ta ble cer si real men te los enun cia dos ha-

10 - Tarsky, A. La concepción semántica de la verdad y los fundamentos de la semántica. Buenos Aires: Nueva Visión, 1972. -Montague, R. "Pragmatics and Intensional logic." En Synthese, 22 (1970), 68-94.

11 - Gadamer, Hans-George. Philosophical Hermeneutica. Berkeley: University of California Press, 1977. -Coreth, E. Cuestiones fundamentales de la Hermenéutica. Barcelona: Herder, 1972. 
blan so bre el mis mo ob je to, bajo el mis mo aspecto y las mismas premisas gnoseológicas. Se puede dar el caso de que aparentemente existe contradicción, pero como se diría en términos hermenéuticos, el contexto es distin to. Aho rabien, si real men te hay con tra dicción entre algunos enunciados, necesariamente uno es verdadero y otro falso, por lo que hay que verificar cuál es el verdadero y de secharelfal so.

\section{CONCLUSIÓN}

Podemos de cir que en la pre sen te ex posición se plantean más preguntas que respuestas, el cam po estáabier to, es pe ran do que se abor den y re suel van las ta reas a rea li zar para la fun da mentación de la Bibliotecología. Dichas tareas han sido sis te ma ti za das y se ha pues to de manifies to la complejidad de esta proble máti ca. La la bor a rea li zar es múl ti ple y cabe mencionar que recae no sólo en los teó ri cos de la disciplina (aunque en ellos principalmente) sino en toda la co mu ni dad de bi blio te có logos; cada miem bro en su cam po debe co la bo rar en su realización. El carácter dialéctico (sistemá ti co) de este tra ba jo per mi te que las ac ciones de cada bibliotecólogoseinterrelacionen entre sí y contribuyan al desarrollo de la Bibliotecología.

\section{BIBLIOGRAFÍA}

Bunge, Mario. Epistemología. Bar ce lo na: Ariel, 1980.275p.

Brown, Harold I. La nueva filosofía de la ciencia. Madrid : Tecnos, 1984. $235 \mathrm{p}$.

Coreth, E. Cuestiones fundamentales de la Hermenéutica. Barcelona : Herder, 1972. 263p.

Chal mers, A.F. ¿Quées esa cosa lla ma da cien cia? Méxi co: Si glo XXI, 1991. $245 p$.

Gadamer, Hans-George. PhilosophicalHermeneutica. Berkeley:University of Ca li for nia Press, 1976. 243p.

Feyerabend, P. K. Tratado contraelméto do. Ma drid: Tec nos, 1981.319 p.

Frege, G. Conceptografía. México: UNAM/IIF, 1972. 270p.

Hanson, N. R. Patterns of discovery. Cambridge : Cambridge University, 1961. 240p.

Kuhn, T.S. Laestructuradelasrevolucionescientíficas. Mé xi co : FCE, 1971. $319 \mathrm{p}$.

Lafuente, R. "La síntesis críticadelconocimientobibliotecológico: su valor para la investigaciónbibliotecológica."En Investigación bibliotecológica. Enero-junio 1988. v. 3, n. 6. p. 3-10.

La ka tos, I. His to ria de la cien ciay sus reconstrucciones racionales. Ma drid: Tecnos, 1974. 158p.

Popper, K. R. El desarrollodelconocimientocientífico: conjeturasy refuta ciones. Bue nos Ai res: Pai dos. 463p.

Popper K. R. Lalógicadela investigacióncientífica. Ma drid: Tec nos, 1973. $451 \mathrm{p}$.

Russell, B. Los problemas de la Filo sofía. México: Nacional, 1975. 187 p.

Russell, B. y Whitehead, A. N. Principia Mathematica. Madrid: Paraninfo, 1981. $471 \mathrm{p}$.

San der, S. "Los ele men tos his tó ri co- teóri cos para la in da ga ción de la es tructura teórica de la Bibliotecología." En Investigación bibliotecológica . Ene ro- ju nio 1989. v. 3, n. 6. p. 31- 37.

Shera, J. Los fundamentos de la educación bibliotecológica. México : UNAM/CUIB, 1990. 520p.

Se quei ra, Or tiz D. y Se quei ra, Or tiz Z. La Bibliote cología comociencia. San José, Costa Rica: Pu bli tex, 1988. 218 p.

Tarsky, A. La con cep ción se mán ti ca de la ver dad y los fun da men tos de la semántica. Buenos Ai res: Nue va Vi sión, 1972.74 p. 